\title{
Numerically Optimized Uniformly Most Powerful Alphabets for Hierarchical-Decode-and-Forward Two-Way Relaying
}

\author{
M. Hekrdla
}

\begin{abstract}
We address the issue of the parametric performance of the Hierarchical-Decode-and-Forward (HDF) strategy in a wireless 2-way relay channel. Promising HDF, representing the concept of wireless network coding, performs well with a pre-coding strategy that requires Channel State Information (CSI) on the transceiver side. Assuming a practical case when CSI is available only on the receiver side and the channel conditions do not allow adaptive strategies, the parametrization causes significant HDF performance degradation for some modulation alphabets. Alphabets that are robust to the parametrization (denoted Uniformly Most Powerful (UMP)) have already been proposed restricting on the class of non-linear multi-dimensional frequency modulations. In this work, we focus on the general design of unrestricted UMP alphabets. We formulate an optimization problem which is solved by standard non-linear convex constrained optimization algorithms, particularly by Nelder-Mead global optimization search, which is further refined by the local interior-points method.
\end{abstract}

Keywords: wireless network coding, hierarchical-decode-and-forward relaying, wireless two-way relay channel, modulation alphabet design, non-linear convex optimization.

\section{Introduction}

Cooperative wireless network protocols which utilize network coding in the physical layer have recently attracted strong research interest due the significant capacity gains that they offer; for citations see [1] and the references therein. The terms Wireless Network Coding (WNC) or Physical-Layer Network Coding (PLNC) are usually used to stress the fact that operations similar to Network Coding (NC) [2] principles are carried out in the wireless domain and completely in the physical layer. Even in a wireless 2-Way Relay Channel (2-WRC), which is the simplest cooperative network, WNC almost doubles the achievable communication sum-rates. 2-WRC consists of two terminals, A and B, bi-directionally communicating with a supporting relay node $\mathrm{R}$ in a half-duplex manner (the terminals cannot send and receive at the same time). In [3] and [4], the authors show that a two-stage DeNoise-and-Forward (DNF)/Hierarchical-Decodeand-Forward (HDF) strategy is very promising in 2 -WRC since it can reliably operate outside the classical Multiple-ACcess (MAC) capacity region [5]. Instead of DNF, we feel that a more generic term $\mathrm{HDF}$ is better suited, especially for more complicated multi-source networks. HDF consists of an MAC stage when A and B are simultaneously transmitting to the relay $\mathrm{R}$ with only hierarchical (exclusively coded) data decoding, and a BroadCast (BC) stage when $\mathrm{R}$ broadcasts the previously-decoded hierarchical data. Exclusive operation allows the termi- nals to decode the hierarchical data using their own data serving as Complementary-Side Information (C-SI) [6]. Assuming canonical linear (one complex dimension; PSK, ASK, QAM) modulation alphabets, HDF strategy suffers from unavoidable wireless channel parametrization in the practical case when only Channel State Information at the Receiver side (CSIR) is available. The problem of parametrization can be suppressed by adaptive extended-cardinality network coding [7]; the adaptation requires moderate channel dynamics, and it is sensitive to channel estimation errors. According to [8], we are able to overcome the parametrization effects by proper multi-dimensional alphabet design. Paper [9] defined a class of Uniformly Most Powerful (UMP) alphabets which are robust to the parametrization. It has been shown that the parametrization is not harmful when the terminals use binary alphabets, but non-binary linear alphabets always suffer from this effect. In this case, more dimensions need to be accommodated to meet the UMP condition. Paper [9] exploits additional dimensions of non-linear multi-dimensional frequency alphabets to fulfill the UMP condition.

In this paper, we study the design of unrestricted multi-dimensional UMP alphabets. We reformulate the UMP alphabet design as a complex optimization problem (maximizing the minimal Euclidean distance constrained by the UMP condition and a unit mean symbol energy constraint) which is to be solved by standard non-linear convex constrained optimization algorithms, particularly by Nelder-Mead global opti- 
mization search, which is further refined by the local interior-points method [10]. The results are presented in Table 1, and the relevant error performance is presented in Sec. 4 .

\section{System Model}

\subsection{Signal space description and used notation}

Let symbol $\mathcal{A}_{T}$ be a modulation alphabet at the terminal $T \in\{A, B\}$. We distinguish two cases: a) both terminals use the same alphabets $\mathcal{A}_{A}=\mathcal{A}_{B}$ or b) the alphabets are assumed to be unequal $\mathcal{A}_{A} \neq \mathcal{A}_{B}$. We assume that both terminals have the same alphabet cardinality $M_{d}=\left|\mathcal{A}_{A}\right|=\left|\mathcal{A}_{B}\right|$ to be a power of two. Further, we do not consider channel coding because it can be serially concatenated with the proposed alphabets, not affecting the results $[4,7]$. The modulation function $\mathcal{M}: \mathbb{Z}_{M_{d}}=\left\{0,1, \ldots M_{d}-1\right\} \rightarrow \mathbb{C}^{N_{s}}$ is assumed to be a memoryless per-symbol mapper, however the output constellation space signals are generally allowed to be multi-dimensional with dimensionality $N_{s}$. Let the mapping correspond directly to the signal indexation, $\mathcal{M}\left(d_{T}\right)=\mathbf{s}_{d_{T}}$, where $d_{T} \in \mathbb{Z}_{M_{d}}$ denotes a data symbol from terminal $T \in\{A, B\}$ and $\mathbf{s}_{T}$ is a multi-dimensional constellation space signal vector.

\subsection{HDF in a wireless two-way relay channel}

The HDF strategy is a two-way relaying concept which consists of two independent stages: MAC and BC. Considering the half-duplex constraint, HDF has the minimum number of stages for two-way relaying. We assume an accurate time synchronized system with full CSIR available (synchronization issues are beyond the scope of our paper) and the channel dynamics does not allow us any adaptation strategies. In the $\mathrm{MAC}$ stage, both $\mathrm{A}$ and $\mathrm{B}$ transmit at the relay in an interfering manner. The received signal is

$$
\mathbf{x}=h_{A} \mathbf{s}_{d_{A}}+h_{B} \mathbf{s}_{d_{B}}+\mathbf{w},
$$

where $\mathbf{w}$ is AWGN with variance $2 N_{0}$ per complex dimension, and the channel parameters $h_{A}$ and $h_{B}$ are complex Gaussian random variables with unit variance for flat Rayleigh fading, and have a Rician distributed envelope with a certain Rician factor for Rice fading. The Rician factor is defined as a power ratio between stationary (Line-Of-Sight) and scattered components. Maximum Likelihood (ML) based processing at $\mathrm{R}$ decodes hierarchical data from the received signal (1) [4], where the hierarchical data is $d_{A B}=\chi\left(d_{A}, d_{B}\right)$; an exclusive operation is a function $\chi: \mathbb{Z}_{M_{d}}^{2} \rightarrow \mathbb{Z}_{M_{d A B}}$, where $M_{d_{A B}}$ denotes the cardinality of exclusive symbol alphabets. The ex- clusive operation meets the exclusive law [7], which guarantees the existence of the invertible function $\chi^{-1}:\left(\mathbb{Z}_{M_{d}}, \mathbb{Z}_{M_{d A B}}\right) \rightarrow \mathbb{Z}_{M_{d}}$. We assume the minimal cardinality exclusive operation $M_{d}=M_{d_{A B}}$, and based on [9] the exclusive operation is fixed on bitwise XOR; $\chi\left(d_{A}, d_{B}\right)=d_{A} \oplus d_{B}$. In the BC stage, $\mathrm{R}$ transmits hierarchical data received previously in the MAC stage; the BC stage is similar to the standard broadcast channel. The terminals decode the desired data from the hierarchical data with the help of its own data and the invertible function, similarly as in NC concepts.

\subsection{UMP alphabets}

It was shown in [9] that the hierarchical minimal Euclidean distance

$$
d_{\min }^{2}(\alpha)=\min _{k \oplus l \neq m \oplus n}\left\|\mathbf{u}_{k l}-\mathbf{u}_{m n}\right\|^{2}
$$

mostly determines the ML-error performance with CSIR [7] and it is parametrized by $\alpha$, where $\mathbf{u}_{i j}=$ $\mathbf{s}_{d_{A}}+\alpha \mathbf{s}_{d_{B}} \in \mathbb{C}^{N_{s}}$ and $\alpha \in \mathbb{C}$ denote a hierarchical signal in the constellation space and a channel parameter, respectively. In the same paper, the authors propose the alphabets and exclusive operations whose minimal distance reaches the upper-bound

$$
d_{\min }^{2}(\alpha) \stackrel{!}{=}|\alpha|^{2} \delta_{\min }^{2}, \forall \alpha \in \mathbb{C},|\alpha| \leq 1,
$$

and they are robust w.r.t. parametrization by $\alpha$; In other words, its performance is parametric, but for all possible parameter values its performance is maximal, therefore such alphabets are denoted as Uniformly Most Powerful (UMP) alphabets. The symbol $\delta_{\min }^{2}$ stands for a minimal distance of the primary alphabet $\mathcal{A}, \delta_{\min }^{2}=\min _{k \neq l}\left\|\mathbf{s}_{k}-\mathbf{s}_{l}\right\|^{2}, k, l \in \mathbb{Z}_{M_{d}}$. The initial definition of the UMP alphabet (3) is equivalent to the following condition [9]

$$
\begin{aligned}
& \left\|\mathbf{s}_{k}-\mathbf{s}_{m}\right\|^{2}+|\alpha|^{2}|| \mathbf{s}_{l}-\mathbf{s}_{n} \|^{2}- \\
& 2|\alpha|\left|\left\langle\mathbf{s}_{k}-\mathbf{s}_{m}, \mathbf{s}_{l}-\mathbf{s}_{n}\right\rangle\right| \geq|\alpha|^{2} \delta_{\min }^{2}
\end{aligned}
$$

for $k \neq m, l \neq n, k \oplus l \neq m \oplus n$ and $\forall \alpha \in \mathbb{C},|\alpha| \leq 1$.

\section{Design of UMP Alphabets}

In contrast to [9], where UMP frequency-modulation alphabets have been designed, we face a problem of general UMP alphabet design with a natural unit mean symbol energy constraint, $\bar{\varepsilon}=\frac{1}{M_{d}} \sum_{i=0}^{M_{d}-1}\left\|\mathbf{s}_{i}\right\|^{2}$. The optimization goal can be formulated as:

$$
\text { maximize } \delta_{\min }^{2}
$$

s. t. $\bar{\varepsilon}=1$ and the UMP condition (4) 
The proposed optimization search has free parameters: alphabet cardinality $M_{d}$ and alphabet dimensionality $N_{s}$. The optimization problem (5) is a standard min-max problem which is conveniently described as

$$
\begin{gathered}
\text { maximize } t \\
\text { s. t. } t \leq\left\|\mathbf{s}_{k}-\mathbf{s}_{l}\right\|^{2}, k \neq l, k, l \in \mathbb{Z}_{M_{d}}, \bar{\varepsilon}=1 \\
\text { and the } \mathrm{UMP} \text { condition (4) }
\end{gathered}
$$

The optimization problem (6) is recognized to be a convex quadratic continuous optimization problem for which standard optimization techniques converge very well, with reasonable precision [10]. Specifically, we have used a Nelder-Mead global-search method with several different starting points of its random number generator, and the results are then refined by the local-search interior-points method. We do not use the direct analytical solution based on the Karush-Kuhn-Tucker conditions due to the large number of constrained conditions (4). We distinguish two cases, a) both terminals use the same alphabet $\mathcal{A}_{A}=\mathcal{A}_{B}$ and case b) $\mathcal{A}_{A} \neq \mathcal{A}_{B}$ where the terminals use unequal alphabets. Case b) needs to incorporate the fact that optimal alphabets should have both minimal distances of $\mathcal{A}_{A}, \mathcal{A}_{B}$ greater than some threshold; therefore we re-define $\delta_{\min }^{2}=\min \left\{\left\|\mathbf{s}_{d_{A}}-\mathbf{s}_{d_{A}^{\prime}}\right\|^{2},\left\|\mathbf{s}_{d_{B}}-\mathbf{s}_{d_{B}^{\prime}}\right\|^{2}\right\}$, where $d_{A} \neq d_{A}^{\prime}, d_{B} \neq d_{B}^{\prime} ; d_{A}, d_{A}^{\prime}, d_{B}, d_{B}^{\prime} \in \mathbb{Z}_{M_{d}}$ to reflect the additional requirement for unequal alphabets.

\section{$3.1 \quad$ Resulting optimized UMP alphabets}

The results are presented in Table 1 . In the third column, maximal attainable minimal distances $\delta_{\text {min }}^{2}$ not constrained by the UMP condition are listed as a reference because they form a natural upper-bound at $\delta_{\min }^{2}$ of UMP alphabets [9]. The 4 th and 5 th columns

\begin{tabular}{|c|c|c|c|c|}
\hline$M_{d}$ & $N_{s}$ & $\delta_{\min }^{2}$, not UMP & $\delta_{\min }^{2}, \mathcal{A}_{A}=\mathcal{A}_{B}$ & $\delta_{\min }^{2}, \mathcal{A}_{A} \neq \mathcal{A}_{B}$ \\
\hline $2^{\dagger)}$ & 1 & 4 & 4 & 4 \\
\hline 4 & $1^{\ddagger)}$ & 2 & 0.4 & 0.68 \\
\hline $4^{\dagger)}$ & 2 & $2 . \overline{6}$ & 2 & 2.18 \\
\hline 4 & 3 & $2 . \overline{6}$ & $2 . \overline{6}$ & $2 . \overline{6}$ \\
\hline
\end{tabular}
contain the obtained UMP alphabets.

Table 1: Numerically optimized UMP alphabets

Alphabets $\dagger$ have the identical spectral efficiency $r=\frac{\log _{2} M_{d}}{N_{s}}=1$, where the extra dimensionality is taken as extra time-slots and a fair mutual comparison by minimal Euclidean distance can be made; here we need to reflect that the modulation should have the same mean symbol energy per timeslot (i.e. with the same mean power), thus in fact the case $\left(M_{d}=4, N_{s}=2, \mathcal{A}_{A}=\mathcal{A}_{B}\right)$, called a 4ary 2D-UMP alphabet, has $\delta_{\min }^{2}=4$, which is directly equal to BPSK, and the alphabet $\left(M_{d}=\right.$ $\left.4, N_{s}=2, \mathcal{A}_{A} \neq \mathcal{A}_{B}\right)$, called a 4ary unequal 2DUMP alphabet, then has $\delta_{\text {min }}^{2}=4.36$, which is better by $10 \log _{10} \frac{4.36}{4} \simeq 0.37 \mathrm{~dB}$ than BPSK, see its hierarchical minimal distance in Figure 2 and the error performance comparison in Fig. 6. Alphabets $\ddagger$ for both $\mathcal{A}_{A}=\mathcal{A}_{B}$ and $\mathcal{A}_{A} \neq \mathcal{A}_{B}$ fulfill the UMP condition only for the channel parameter $|\alpha|=1$ (we call these weak-UMP alphabets), since 1D-UMP alphabets must be binary only [9]. The optimal alphabet for $\left(M_{d}=4, N_{s}=1, \mathcal{A}_{A}=\mathcal{A}_{B}\right)$ : $\mathcal{A}=\left\{-2 \sqrt{\frac{2}{5}},-\sqrt{\frac{2}{5}}, \sqrt{\frac{2}{5}}, 2 \sqrt{\frac{2}{5}}\right\}$, see the constellation in Figure 3 and its hierarchical minimal distance depicted in Figure 1, surprisingly resembles 4-ASK

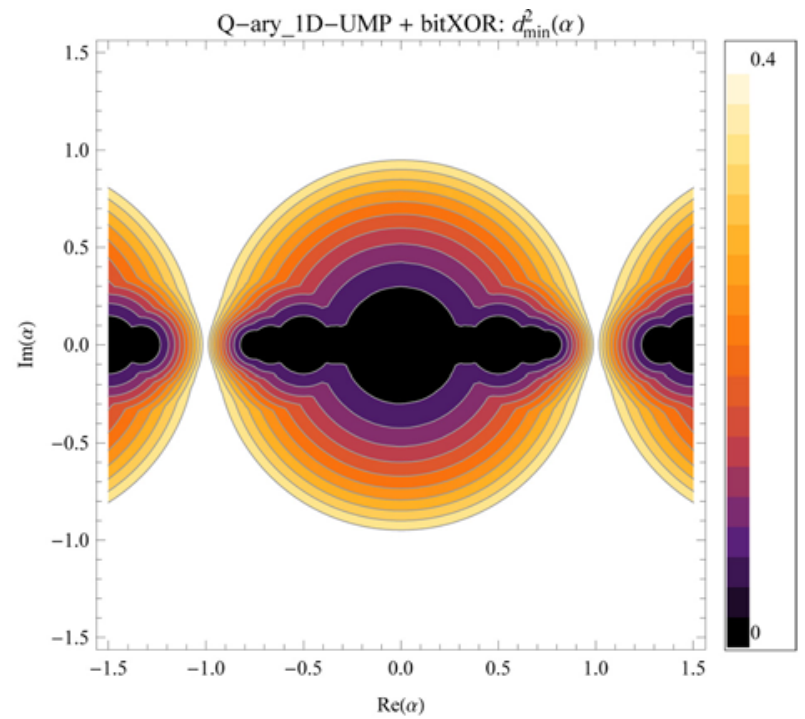

Fig. 1: Parametric hierarchical minimal distance of quaternary 1D-weak UMP with equal alphabets

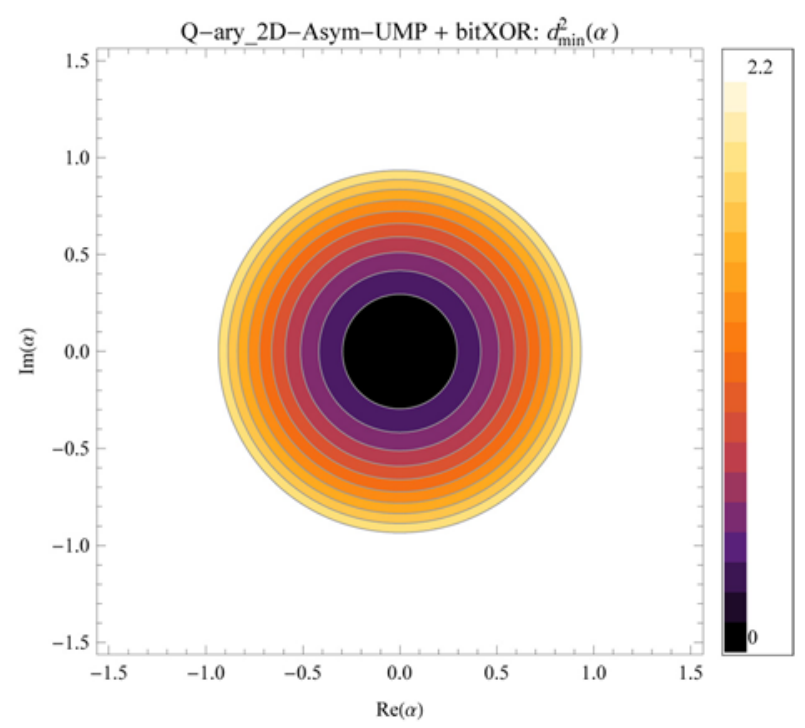

Fig. 2: Parametric hierarchical minimal distance of quaternary 2D-UMP with equal alphabets 


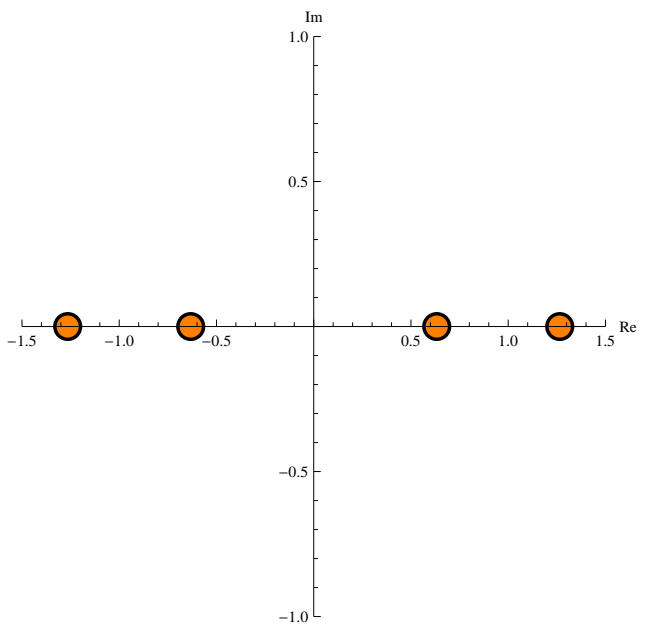

Fig. 3: The constellation of 4ary 1D-UMP with equal alphabets resembles standard 4-ASK modulation

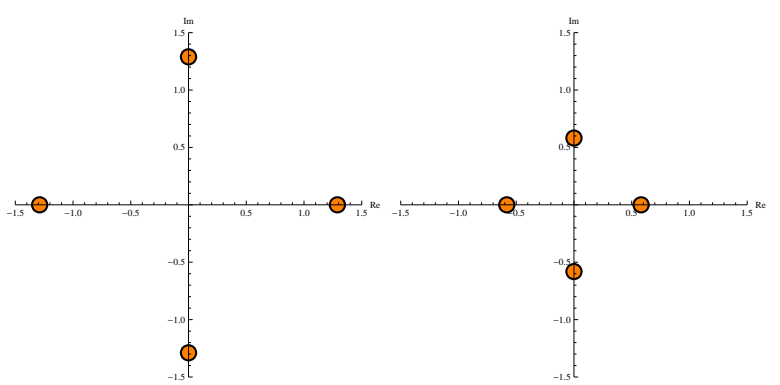

Fig. 4: The constellations of quaternary 1D-UMP with unequal alphabets, alphabets $\mathcal{A}_{A}$ and $\mathcal{A}_{B}$, respectively, resembles the scaled versions of standard QPSK constellations

modulation: $\mathcal{A}=\left\{-\frac{3}{\sqrt{5}},-\frac{1}{\sqrt{5}}, \frac{1}{\sqrt{5}}, \frac{3}{\sqrt{5}}\right\}$. The other optimal case $\ddagger$ with $\left(M_{d}=4, N_{S}=1\right)$ and $\mathcal{A}_{A} \neq \mathcal{A}_{B}$ astonishingly seems to correspond to the scaled versions of two QPSK constellations, see Figure 4.

\section{Performance Evaluation}

In this section, we compare the Symbol Error Rate (SER) of quaternary 1D alphabets: QPSK, 4-ASK and the proposed 4ary 1D-UMP alphabets with equal and unequal alphabets $\ddagger$. The error performance in Figure 5 is in accordance with our expectations. In the Rayleigh fading channel the UMP condition is not so interesting, rather the overall $\delta_{\min }^{2}$ is determining. In contrast to Rayleigh fading, it is preferable to use UMP alphabets even at the price of lower $\delta_{\min }^{2}$ in the Rician fading channel (here the Rician factor $K=10 \mathrm{~dB}$ ). A remarkable observation is that for sufficiently strong SNR the performance of 4ary 1D-UMP for equal and unequal alphabets is almost identical.

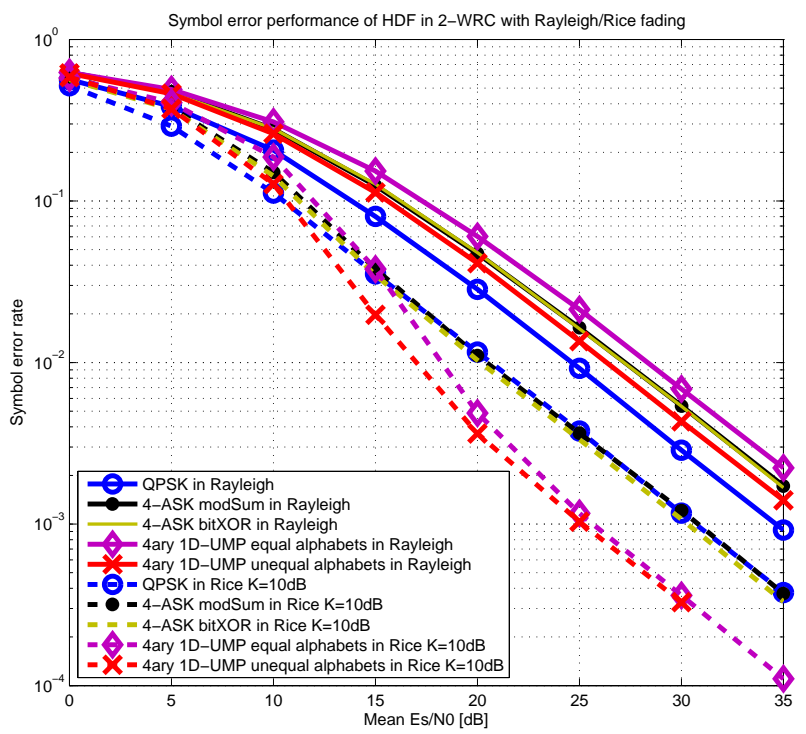

Fig. 5: Symbol error rate of quaternary alphabets with a single complex dimension

Bit throughput is a relevant performance measure for alphabets with the same spectral efficiency $\dagger$. In Figure 6 we compare BPSK with Quaternary 2DUMP alphabets with the case of equal and unequal alphabets. As expected, the quaternary unequal 2DUMP alphabet outperforms BPSK by $\sim 0.5 \mathrm{~dB}$. At the cost of lower spectral efficiency, 4ary 3D-UMP has better error performance.

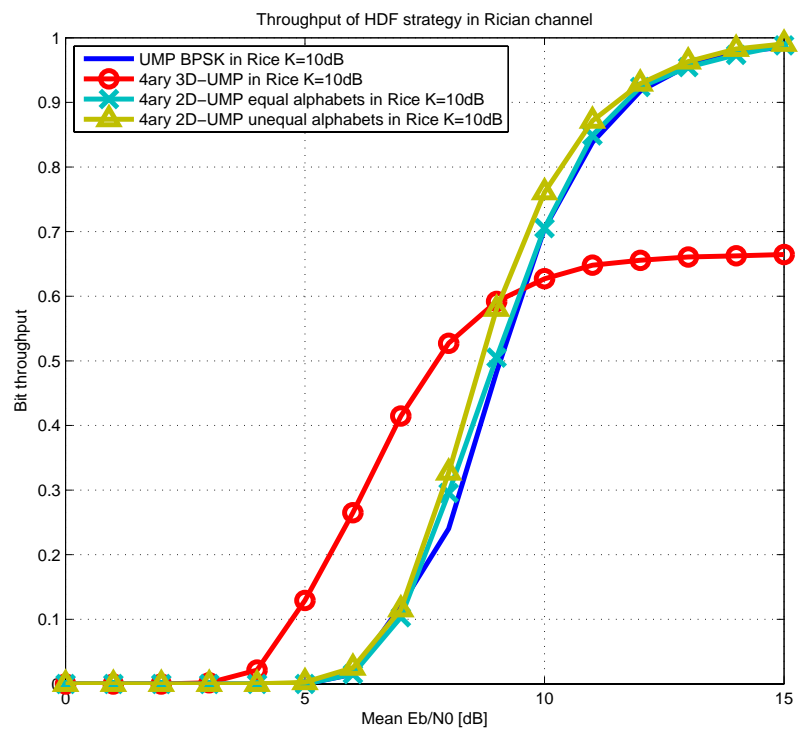

Fig. 6: Bit throughput of the proposed alphabets of HDF strategy in Rice fading with Rician factor $K=10 \mathrm{~dB}$

\section{Conclusions and Discussion}

We have applied canonical convex constrained nonlinear optimization methods for general unrestricted design of Uniformly Most Powerful (UMP) alphabets, 
which are convenient for the Hierarchical-Decodeand-Forward (HDF) strategy in a wireless 2-way relay channel with Channel State Information (CSI) on the receiver side, where the channel conditions do not allow adaptive strategies. UMP alphabets that are robust to parametrization (denoted Uniformly Most Powerful (UMP)) have already been proposed restricting on non-linear multi-dimensional frequency modulations. We have re-formulated the optimization problem and solved by the Nelder-Mead global optimization method, which provided starting points to the interior-points method. The proposed nonbinary linear (one dimensional) alphabets outperform canonical linear alphabets (PSK, ASK, QAM), and the proposed multi-dimensional alphabets outperform UMP-BPSK modulation. Utilization of the proposed alphabets with suitable mappings on the transitions of convolutional codes (similarly to the concept of trellis coded modulation), possibly further serially concatenated with an inner code forming a turbo coded system, would be preferable in a practical design. We have supported the idea that unequal alphabets which are made as a power-scaled version of canonical linear modulations are well robust to the parametrization problem. This work may serve as a basis for multi-dimensional Multiple-Input MultipleOutput (MIMO)-UMP alphabet design, which will be a topic for future work.

\section{Acknowledgement}

This work was supported by the FP7-ICT SAPHYRE project; by Grant Agency of the Czech Republic, project 102/09/1624; and by the Grant Agency of the Czech Technical University in Prague, grant No. SGS10/287/OHK3/3T/13.

\section{About the author}

Miroslav Hekrdla received his M.Sc. degree in Electrical Engineering from the Czech Technical University in Prague, Czech Republic, in 2009. His research interests include nonlinear space-time modulation, coding and processing, relay-based wireless communication systems with distributed, cooperative and MIMO processing, and aspects of information theory and channel coding.

\section{References}

[1] Fu, S., Lu, K., Zhang, T., Qian, Y., Chen, H.-H.: Cooperative wireless networks based on physical layer network coding, Wireless Communications, IEEE, vol. 17, no. 6, 2010, pp. 86-95.
[2] Yeung, R. W., Li, S.-Y. R., Cai, N., Zhang, Z.: Network Coding Theory. now Publishers, 2006.

[3] Popovski, P., Yomo, H.: Physical network coding in two-way wireless relay channels, In Proc. IEEE Internat. Conf. on Commun. (ICC), jun. 2007, pp. 707-712.

[4] Sykora, J., Burr, A.: Hierarchical alphabet and parametric channel constrained capacity regions for $\mathrm{HDF}$ strategy in parametric wireless 2-WRC, In Proc. IEEE Wireless Commun. Network. Conf. (WCNC), (Sydney, Australia), apr 2010, pp. 1-6.

[5] Cover, T. M., Thomas, J. A.: Elements of Information Theory. John Wiley \& Sons, 1991.

[6] Sykora, J., Burr, A.: Network coded modulation with partial sideinformation and hierarchical decode and forward relay sharing in multisource wireless network, In Wireless Conference $(E W)$, 2010 European, 2010, pp. 639-645.

[7] Koike-Akino, T., Popovski, P., Tarokh, V.: Optimized constellations for two-way wireless relaying with physical network coding, Selected Areas in Communications, IEEE Journal on, vol. 27, jun. 2009, pp. 773-787.

[8] Uricar, T., Sykora, J.: Design criteria for hierarchical exclusive code with parameter-invariant decision regions for wireless 2-way relay channel, EURASIP Journal on Wireless Communications and Networking, vol. 2010, 2010, pp. 1-13. Article ID 921427.

[9] Hekrdla, M., Sykora, J.: Channel parameter invariant network coded FSK modulation for hierarchical decode and forward strategy in wireless 2-way relay channel, In COST $2100 \mathrm{MCM}$, (Aalborg, Denmark), June 2010, pp. 1-8. TD10-11087.

[10] Boyd, S., Vandenberghe, L.: Convex Optimization. New York, NY, USA : Cambridge University Press, 2004.

Miroslav Hekrdla

E-mail: miroslav.hekrdla@fel.cvut.cz Department of Radio Engineering Faculty of Electrical Engineering Czech Technical University in Prague Technická 2, 16627 Prauge, Czech Republic 